\title{
AVALIAÇÃO DA QUALIDADE FISIOLÓGICA E SANITÁRIA DE SEMENTES DE GENÓTIPOS DE SOJA DO CICLO PRECOCE/MÉDIO EM UBERLÂNDIA, MINAS GERAIS
}

\author{
OSVALDO T. HAMAWAKI ${ }^{1}$, FERNANDO C. JULIATTI ${ }^{1}$, GERALDO M. GOMES ${ }^{1}$, \\ FABRÍCIO A. RODRIGUES ${ }^{2} \&$ VERA LÚCIA M. SANTOS
}

\begin{abstract}
${ }^{1}$ Instituto de Ciências Agrárias, Universidade Federal de Uberlândia, Campus Umuarama, Cx. Postal 593, CEP 38400-902, Uberlândia, MG, e-mail: hamawaki@umuarama.ufu.br, juliatti@ufu.br; ${ }^{2}$ Plant Pathology Department, University of Florida, 32611-0680, Gainesville, FL, USA
\end{abstract}

(Aceito para publicação em 31/10/2001)

Autor para correspondência: Fernando Cezar Juliatti

HAMAWAKI, O.T., JULIATTI, F.C., GOMES, G.M \& RODRIGUES, F.A. Avaliação da qualidade fisiológica e sanitária de sementes de genótipos de soja do ciclo precoce/médio Uberlândia, Minas Gerais. Fitopatologia Brasileira 27:201-205. 2002.

\section{RESUMO}

Este trabalho teve como objetivo avaliar porcentagem de germinação, vigor, sanidade de sementes e produtividade de 18 linhagens de soja (Glycine max). O ensaio de campo foi conduzido na Fazenda Experimental do Capim Branco da Universidade Federal de Uberlândia. Utilizou-se o delineamento em blocos casualizados, com quatro repetições e 18 tratamentos, constituídos pelos genótipos de soja. Os genótipos UFV94-426803 e BR9206665 apresentaram a maior e a menor porcentagem de germinação (96 e 69\%, respectivamente). Essa variável correlacionou-se positivamente com o vigor das sementes $(\mathrm{r}=+0,79)$, e negativamente com a incidência de Fusarium semitectum, Phomopsis sojae e Colletotrichum dematium ( $\mathrm{r}=-0,33,-0,39, \mathrm{e}-0,26$, respectivamente). As linhagens CSP-5 e BR93-6957 apresentaram, respectivamente, o maior (90) e o menor $(54,5)$ vigor das sementes. Houve correlação positiva do vigor das sementes com a produtividade de grãos $(r=$
$+0,59)$, e negativa com a incidência de $F$. semitectum e $P$. sojae $(\mathrm{r}=$ $-0,36$ e $-0,60$, respectivamente). Verificou-se uma incidência de 47 e $9 \%$ de F. semitectum, respectivamente, para os genótipos FT-2002 e CSP-5. Os genótipos BR93-6957 e UFV94-426803 apresentaram, respectivamente, 49,7 e $2,1 \%$ de incidência de $P$. sojae e 'CSP-2' foi o genótipo que apresentou a maior incidência de $C$. dematium, (3,8\%). 'FT-2001' e 'UFV94-1896' apresentaram, respectivamente, a maior $(3,9 \mathrm{t} / \mathrm{ha})$ e a menor $(2,48 \mathrm{t} / \mathrm{ha})$ produtividade de grãos. Houve correlação negativa dessa variável com a incidência de $P$. sojae $(\mathrm{r}=-0,44)$. As cultivares BR-16 e OCEPAR-3 apresentaram valores intermediários para todas variáveis avaliadas, embora não tenham diferido significativamente dos demais genótipos avaliados nesse ensaio.

Palavras-chave adicionais: sanidade de sementes, fungos, incidência, cultivares e produtividade.

\begin{abstract}
Evaliation of the physiological and health quality of genotipe of soybean genotype seeds in Uberlândia, Minas Gerais

The objective of this work was to evaluate the percentage of variables in 18 lines of soybean (Glycine max) in terms of germination, seed vigour, health and productivity. The experimental design utilized consisted of randon blocks, with four replications, and 18 treatments, according to soybean genotypes. The genotypes UFV94-426803 e BR92-06665 showed the longest and shortest germination times, 96 and 69\%, respectively. This variable showed a positive correlation with seed vigour $(\mathrm{r}=+0.79)$, and a negative correlation with the incidence of Fusarium semitectum, Phomopsis sojae e Colletotrichum dematium. ( $\mathrm{r}=-0.33,-0.39$, e -0.26 , respectively). In terms of seed vigour, the lines CSP-5 e BR93-6957 represented the highest and lowest percentages, 90 and $54.5 \%$, respectively. There was a positive correlation between seed vigour and grain

productivity $(\mathrm{r}=+0.59)$ and a negative correlation between seed vigour and the incidence of $F$. semitectum and $P$. sojae $(\mathrm{r}=-$ $0.36 \mathrm{e}-0.60$, respectively). The incidence of $F$. semitectum was $47 \%$ in the FT-2002 line and 9\% in the CSP-5 line. The incidence of $P$. sojae in the genotypes BR93-6957 and UFV94426803 was 49.7 and $2.1 \%$, respectively. The CSP line presented the highest incidence of $C$. dematium (3.8\%). The genotypes FT-2001 and UFV94-1896 showed the highest (3.9 t/ha) and the lowest $(2.48 \mathrm{t} / \mathrm{ha})$ grain productivity. There was a negative correlation between this variable and the incidence of $P$. sojae $(\mathrm{r}=-0.44)$. The cultivars BR-16 e OCEPAR-3 presented intermediated values for all variables, but did not show smaller yields (grain production) than the other genotypes available in this experiment.
\end{abstract}

A qualidade fisiológica e sanitária de sementes de soja [Glycine max (L.) Merril] é fator de importância no estabele- cimento de uma lavoura. A baixa qualidade fisiológica, no que se refere ao poder de germinação e vigor das sementes, 


\section{O.T. Hamawaki et al.}

produzidas na maioria das áreas situadas ao norte do paralelo $24^{\circ} \mathrm{LS}$, tem sido um dos principais problemas para a cultura nessas regiões (Arantes et al., 1994).

As características mais importantes da semente de soja são a ausência de rachaduras no tegumento, alta porcentagem de germinação e o vigor (Sediyama et al., 1985). A ocorrência de doenças é outro fator limitante para a obtenção de alto rendimento na produção de grãos. Com a expansão da cultura de soja na Região Central do Brasil, a falta de tratamento químico das sementes acarretou o aparecimento de inúmeras doenças antes consideradas de menor importância (Yorinori et al., 1993). A antracnose e a seca da haste e da vagem têm atingido proporções de epidêmicas nas regiões mais quentes e úmidas dos cerrados onde a temperatura é mais elevada e as chuvas são mais intensas e freqüentes. Os patógenos Colletotrihcum dematium var. truncata (Schw.) Von Arx, Phomopsis sojae Lehman, Cercospora kikuchii (Matsu. \& Tomoy) Gardner, Cercospora sojina Hara e Septoria glycines Hemmi, são os mais freqüentemente disseminados através das sementes.

Cultivares de ciclos diferentes numa mesma região apresentam, de um modo geral, grande variação quanto à qualidade das sementes (Yorinori et al., 1986). Variedades de soja cultivadas em temperaturas, com média anual acima de $30^{\circ} \mathrm{C}$, e chuvas no período da maturação à colheita, são predispostas a infecções por $P$. sojae, C. dematium var. truncata e Fusarium semitectum Berk \& Rav. Geralmente, as cultivares precoces e de ciclo médio, as quais findam seus ciclos entre meados de fevereiro e março, estão sujeitas a altas temperaturas e umidade, e desta forma podem ser infetadas por tais patógenos, em comparação às cultivares tardias. Sementes obtidas de campos produtores sujeitos a tais condições climáticas poderão ser descartadas, até mesmo antes da análise sanitária e fisiológica.

Este trabalho teve como objetivo avaliar a qualidade fisiológica e sanitária das sementes de genótipos de soja nas condições edafo-climáticas de Uberlândia, Minas Gerais e verificar a possibilidade da aplicação do teste de sanidade como indicador da produtividade de linhagens de soja.

O ensaio de campo foi conduzido na Fazenda Experimental do Capim Branco, pertencente à Universidade Federal de Uberlândia, localizada no município de Uberlândia, MG, a qual situa-se na latitude $18^{\circ} 55^{\prime} 23^{\prime}$ 'S, longitude $48^{\circ} 17^{\prime} 19^{\prime}$ 'W e altitude de $872 \mathrm{~m}$. A precipitação pluvial média anual registrada nesta localidade é de $1.250 \mathrm{~mm}$, sendo o solo da área experimental pertencente à classe Latossolo Vermelho-escuro, textura média. O preparo do solo, a adubação de plantio e todos os tratos culturais foram realizados de acordo com as recomendações técnicas para a cultura da soja. Na safra agrícola 1996/97, as linhagens BR92-06665, BR93-4854, BR93-6957, BR93-7411, BR93-11995, CSP-1, CSP-2, CSP-3, CSP-4, CSP-5, FT-20, FT-2001, FT-2002, T2003, UFV89-334430, UFV94-1896, UFV94-426796, UFV94-426803 e as cultivares BR-16 e OCEPAR-3 do ensaio final de soja precoce/médio de Minas Gerais foram semeadas em parcelas de quatro fileiras de $5 \mathrm{~m}$ de comprimento, espaçadas de $0,50 \mathrm{~m}$ e densidade de 18 a 20 plantas por metro linear. Utilizou-se o delineamento em blocos casualizados, com três repetições e 18 tratamentos, constituídos pelos genótipos de soja. A parcela útil foi constituída das duas linhas centrais de cada parcela, eliminando-se $0,5 \mathrm{~m}$ de cada extremidade, resultando em uma área de $4 \mathrm{~m}^{2}$.

Colheram-se todas as plantas presentes na área útil das parcelas, determinando-se, após secagem uniforme até $12 \%$ de umidade, a produtividade de grãos. As sementes obtidas de cada parcela e referentes à cada tratamento, foram acondicionadas em envelopes de papel pardo, colocadas em caixas de arquivo morto e armazenadas em câmara fria (temperatura de $15^{\circ} \mathrm{C}$ e umidade relativa do ar de $47 \%$ ), até a realização dos testes fisiológicos e de sanidade. Quando da realização dos testes, as sementes foram homogeneizadas e, utilizando-se um divisor de amostras (modelo Borner), retirou-se 200 sementes de cada genótipo de soja para os testes de germinação e vigor, e 400 sementes para o teste de sanidade (quatro repetições de 100 sementes).

As variáveis avaliadas na determinação da qualidade fisiológica das sementes foram: a) grau de umidade: pelo uso de um determinador de umidade, modelo GEOLE 400. Utilizaram-se duas repetições, conforme recomenda a Regra de Análise de Sementes (Brasil, 1992), e, em média, 142 g de sementes por amostra, armazenada à temperatura ambiente. As condições de armazenamento, em média, a 21 ${ }^{\circ} \mathrm{C}$, determinando um acréscimo de $0,5 \%$ em cada leitura determinada diretamente no visor do aparelho; b) peso de 1.000 sementes (oito sub-amostras de 100 sementes/parcela). As sementes foram contadas com auxílio de um tabuleiro contador, calculando-se os coeficientes de variação dos dados obtidos nas pesagens de cada parcela, os quais não excederam a 4\% (Brasil, 1992). De posse dos valores obtidos nas pesagens, efetuou-se a correção dos pesos em função do grau de umidade, considerando-se o grau de umidade igual a 10\%, através da aplicação da fórmula: Pf $=$ Pi (100 - Ui $)$ / (100-Uf) onde: $\mathrm{Pf}=$ peso final da amostra, $\mathrm{Pi}=$ peso inicial da amostra, Uf $=$ umidade final da amostra e Ui $=$ umidade inicial da amostra; c) testes de germinação e vigor: as amostras foram submetidas ao teste de germinação e classificação do vigor das plântulas, utilizando-se 200 sementes por lote, perfazendo-se quatro sub-amostras de 50 sementes para cada parcela, conforme recomendado por Santos (1994). As sementes foram colocadas para germinar em papel Germitest, previamente umedecido com água desmineralizada, confeccionando-se os rolos. A quantidade de água em mililitro utilizada para umedecer as folhas de papel foi de duas e meia o peso do papel seco em gramas. Após a montagem, os rolos foram colocados em um germinador tipo Mangelsdorf, à temperatura constante de $25^{\circ} \mathrm{C}$. As avaliações das plântulas normais e anormais foram realizadas aos cinco e aos oito dias após a montagem do teste, conforme especificado nas Regras para Análise de Sementes (Brasil, 1992). As sementes normais foram classificadas quanto ao vigor, nas categorias fortes ou fracas. Foi considerado como plântulas normais, na primeira 
contagem, aquelas que apresentavam as partes morfológicas completas e sem lesões e alcançavam o padrão determinado para o genótipo. Na segunda contagem, aos oito dias, todas as plântulas remanescentes foram classificadas como normais ou anormais. As Plântulas classificadas como fracas foram as que se apresentaram abaixo do padrão, como: raízes mal formadas, plântula comprida, fina, mal desenvolvida ou raquítica, para o padrão estabelecido para o genótipo avaliado. Foram classificadas como anormais danificadas as plântulas que apresentaram algum tipo de dano, causado pelo ataque de insetos, danos mecânicos, ou pela umidade. Aquelas que apresentaram-se seriamente deterioradas devido à presença de microrganismos patogênicos, prejudicando o seu desenvolvimento normal, presente na radícula, hipocótilo, cotilédones ou na plúmula foram consideradas infeccionadas Sementes mortas: foram contadas como sementes mortas, aquelas que se encontraram amolecidas e em estado de decomposição. A maioria foi retirada e contada na primeira leitura, aos cinco dias, para evitar que prejudicassem o desenvolvimento das outras.

O teste de sanidade de sementes foi realizado utilizandose o método do papel de filtro "Blotter test". As sementes foram colocadas em gerbox previamente desinfestado com hipoclorito de sódio a $2 \%$ e álcool absoluto ( $\left.96^{\circ} \mathrm{GL}\right)$. O substrato foi composto de duas folhas de papel toalha, sobre as quais colocou-se uma folha de papel de filtro. Essas folhas foram previamente esterilizadas em autoclave durante $20 \mathrm{~min}$, à temperatura de $120^{\circ} \mathrm{C}$ e pressão variando de 1,3 a $1,5 \mathrm{~atm}$ .O substrato foi umedecido com água destilada e autoclavada, na quantidade de $15 \mathrm{ml}$ por gerbox, o que foi suficiente para promover a umidade necessária ao desenvolvimento dos possíveis fitopatógenos presentes nas sementes. As sementes foram incubadas durante oito dias, sob fotoperíodo de $12 \mathrm{~h}$ de luz artificial e $12 \mathrm{~h}$ no escuro e temperatura variando de 20 a $22{ }^{\circ} \mathrm{C}$. Utilizaram-se 25 sementes de soja em cada gerbox, totalizando, assim, 100 sementes por genótipo. Foi adotado o delineamento experimental inteiramente casualizado, com quatro repetições e 18 tratamentos. A identificação dos fitopatógenos foi realizada com o auxilio de um microscópio 40x, e quando necessário confirmar a presença de suas estruturas, utilizou-se o microscópio composto 400x. Atentou-se para a presença dos fungos $F$. semitectum, P. sojae, C. kikuchii, C. sojina, C. dematium, Rhizoctonia solani Kühn e Macrophomina phaseolina (Tassi) Goid de forma a atender as Regras para Análise de Sementes (Brasil,1992).

Os dados obtidos, referente à cada variável avaliada, foram submetidos à análise de variância, pelo teste $\mathrm{F}$, e comparação das médias pelo teste de Tukey, ao nível de 5\% de probabilidade. A porcentagem de incidência dos patógenos determinados nas amostras foi transformada para arco seno da raiz de x/100, segundo Banzato \& Kronka (1992).

Os valores de porcentagem de germinação e vigor das sementes para as cultivares BR-16 e OCEPAR-3, consideradas como testemunhas, e para as 18 linhagens estudadas estão representados na Tabela 1. Observa-se que a linhagem UFV94-426803 apresentou a maior porcentagem de germinação (90\%), enquanto que 'BR92-06665' obteve o menor valor dessa variável (69\%). Apenas 'BR92-06665' apresentou o nível mínimo de germinação recomendado pela Comissão Estadual de Sementes de Minas Gerais, o qual é de $75 \%$ (Minas Gerais, 1997). Com relação ao vigor das sementes, os genótipos CSP-5 e BR93-6957 obtiveram, respectivamente, o maior $(90 \%)$ e o menor $(55 \%)$ valor dessa variável (Tabela 1). O fungo $P$. sojae é considerado o principal patógeno relacionado com a qualidade das sementes de soja, o qual perde rapidamente a sua agressividade durante o armazenamento realizado sob condição de ambiente natural, ocorrendo, ao mesmo tempo, aumento gradual na germinação das sementes em laboratório (Henning, 1987). Segundo o autor, no final de três meses de armazenamento, ocorreu uma queda de $50 \%$ na incidência de $P$. sojae A porcentagem de sementes contaminadas pela microflora fúngica decresce com o aumento no período de armazenamento. Com 13 meses ou mais, os fungos freqüentemente encontrados no campo já não estão mais presentes nas sementes colhidas (Resende \& Sediyama, 1995).

Com relação à variável produtividade de grãos, observa-se, que a linhagem FT-2001 obteve a maior média (3,9 t/ha) (Tabela 1), já o menor valor foi verificado para a linhagem UFV94-1896 com 2,5 t/ha, com diferença estatística entre elas. Houve também diferença significativa entre as testemunhas 'BR-16' e 'OCEPAR-3' com 3,1 e 2,7

TABELA 1 - Porcentagem de germminação, vigor e produtividade de grãos dos 20 genótipos de soja (Glicine max) avaliados. Uberlândia, MG, 1996/97

\begin{tabular}{|c|c|c|c|}
\hline \multirow[b]{2}{*}{ Genótipo } & \multicolumn{3}{|c|}{ Variáveis* } \\
\hline & $\begin{array}{c}\text { Germinação } \\
(\%)\end{array}$ & $\begin{array}{l}\text { Vigor } \\
(\%)\end{array}$ & $\begin{array}{c}\text { Produtividade } \\
\text { (t/ha) }\end{array}$ \\
\hline UVF94-426803 & $96,0 \mathrm{a}$ & $89,5 \mathrm{a}$ & $3,1 \mathrm{abc}$ \\
\hline CSP-5 & $95,0 \mathrm{a}$ & $90,0 \mathrm{a}$ & $3,4 \mathrm{abc}$ \\
\hline FT-2001 & $92,0 \mathrm{ab}$ & $85,5 \mathrm{ab}$ & $3,9 \mathrm{a}$ \\
\hline CSP-1 & $90,5 \mathrm{ab}$ & 80,0 abc & $3,5 \mathrm{ab}$ \\
\hline CSP-4 & $89,0 \mathrm{ab}$ & 76,0 abc & $3,2 \mathrm{abc}$ \\
\hline FT-2002 & $88,5 \mathrm{ab}$ & 68,5 bcd & $2,9 \mathrm{abc}$ \\
\hline BR93-4854 & $88,5 \mathrm{ab}$ & $84,0 \mathrm{ab}$ & $3,4 \mathrm{abc}$ \\
\hline CSP-3 & $87,5 \mathrm{ab}$ & $78,5 \mathrm{abc}$ & $3,3 \mathrm{abc}$ \\
\hline UFV94-426796 & 86,5 abc & $80,0 \mathrm{abc}$ & $3,1 \mathrm{abc}$ \\
\hline UFV94-1896 & $86,0 \mathrm{abc}$ & 71,5 abcd & $2,5 \quad \mathrm{c}$ \\
\hline BR-16 & 86,0 abc & 73,0 abcd & $3,1 \mathrm{abc}$ \\
\hline BR93-7411 & $86,0 \mathrm{abc}$ & $75,5 \mathrm{abc}$ & $3,4 \mathrm{abc}$ \\
\hline FT-2003 & 81,0 abc & 72,5 abcd & $2,9 \mathrm{abc}$ \\
\hline CSP-2 & $81,0 \mathrm{abc}$ & $68,5 \mathrm{bcd}$ & $3,5 \mathrm{ab}$ \\
\hline BR93-6957 & $80,5 \mathrm{abc}$ & 54,5 & $2,7 \mathrm{bc}$ \\
\hline OCEPAR-3 & $80,5 \mathrm{abc}$ & 71,5 abcd & $2,7 \mathrm{bc}$ \\
\hline UFV89-334430 & $80,5 \mathrm{abc}$ & 70,0 abcd & $2,8 \mathrm{bc}$ \\
\hline BR93-11995 & $80,0 \mathrm{abc}$ & $71,5 \mathrm{abcd}$ & $3,4 \mathrm{abc}$ \\
\hline FT-20 & 75,0 bc & $62,5 \mathrm{~cd}$ & $2,8 \mathrm{bc}$ \\
\hline BR92-06665 & $68,5 \quad \mathrm{c}$ & $60,5 \mathrm{~cd}$ & $2,7 \mathrm{bc}$ \\
\hline
\end{tabular}

*Médias seguidas pela mesma letra não diferem entre si, ao nível de 5\% de probabilidade pelo teste de Tukey 


\section{O.T. Hamawaki et al.}

t/ha, respectivamente. Em comparação aos demais genótipos, a testemunha 'BR-16' não diferiu significativamente daqueles apresentando as maiores médias, enquanto que 'OCEPAR-3' apresentou diferença significativa em relação aos genótipos com as maiores médias de produtividade (Tabela 1). A produtividade de grãos para todos os genótipos avaliados pode ser considerada alta. Como exemplo, o genótipo que apresentou um desempenho inferior aos demais ('UFV94-1896' com 2,5 t/ha) posicionou-se acima da média nacional que é de 2,1 t/ha. Santos (1994) obteve resultados semelhantes para as cultivares Doko e FT-11, para as quais a produtividade foi de $3,4 \mathrm{t} / \mathrm{ha}$.

Em decorrência da baixa incidência de $M$. phaseolina, C. kikuchii, C. sojina e R.. solani, os dados não foram analisados. Observa-se que o genótipo FT-20 apresentou a maior incidência de $F$. semitectum $(35,17 \%)$, e 'FT-2OO1' a menor incidência (6,55\%) (Tabela 2). Para as duas testemunhas, OCEPAR-3 e BR-16, a incidência desse patógeno foi de $17,47 \%$ e $9,20 \%$, respectivamente, com diferença significativa entre elas. A incidência de $F$. semitectum nas sementes dos genótipos testados podem ser considerada baixa, quando comparada com a incidência verificada nas sementes das cultivares Cristalina (52\%), CAC-1 $(42,8 \%)$ e Savana (40,9\%), conforme encontrado por Souza (1995). É comum a associação entre F. semitectum e $R$. solani em sementes de soja, já que esses dois fungos são habitantes naturais do solo e causam juntos sérios problemas em algumas áreas de plantio de soja na fase inicial de estabelecimento da cultura.

Com relação à incidência de $P$. sojae verifica-se que os genótipos BR93-6957 e UFV94-426803 apresentaram, respectivamente, a maior $(48,73 \%)$ e a menor $(2,06 \%)$ incidência do fungo (Tabela 2). Nas duas testemunhas, a maior incidência de $P$. sojae foi verificado para 'OCEPAR3' (46,45\%), e a menor incidência para 'BR-16' (3,12\%), com diferença significativa entre elas. A seca ou queima das hastes e das vagens, causada por $P$. sojae, é comum em nossas lavouras de soja, prevalecendo em épocas quentes e chuvosas, além de estar comumente associada à antracnose. Essa doença é responsável pelo descarte de um grande número de lotes de sementes. Cultivares precoces, como é o caso dos genótipos selecionados para esse ensaio, com maturação ocorrendo no período chuvoso, são severamente acometidas por doenças. Um estudo realizado por Souza (1995), objetivando determinar a sanidade das sementes produzidas em lavouras de soja do município de Nova Ponte (MG), revelou que a cultivar IAC-8 foi a que apresentou a maior incidência de $P$. sojae (26,5\%), enquanto que as cultivares UFV-10, CAC-1 e Savana apresentaram a menor incidência do patógeno $(0,78 ; 0,50$ e $1,94 \%$, respectivamente), as quais posicionaram-se abaixo da porcentagem de incidência para descarte de um lote.

Observa-se que o genótipo CSP-2 apresentou a maior incidência de $C$. dematium $(3,10 \%)$, enquanto que para os genótipos UFV94-426796 e FT-2001 não foi observada a ocorrência do fungo (Tabela 2). As testemunhas 'OCEPAR3' e 'BR-16' apresentaram, respectivamente, $0,47 \%$ e $0,06 \%$ de incidência do fungo e não diferiram significativamente entre si, contudo diferiram significativamente dos genótipos 'CSP-2', 'UFV94-426796' e 'FT-2001'. Souza (1995), verifi-

TABELA 2 - Porcentagem de incidência de Fusarium semitectum, Phomopsis sojae e Colletotrichum dematium nas sementes dos 20 genótipos de soja (Glycine max) avaliados. Uberlândia, MG, 1996/97

\begin{tabular}{|c|c|c|c|c|c|}
\hline Genótipo & $\begin{array}{c}\text { Fusarium } \\
\text { semitectum }^{*}\end{array}$ & Genótipo & $\begin{array}{c}\text { Phomopsis } \\
\text { sojae }\end{array}$ & Genótipo & $\begin{array}{c}\text { Colletotrichum } \\
\text { dematium }^{*}\end{array}$ \\
\hline FT-20 & $35,17 \mathrm{a}$ & BR93-6957 & $49,73 a$ & CSP-2 & $3,84 a$ \\
\hline FT-2002 & $29,69 a$ & OCEPAR-3 & $46,45^{\mathrm{a} b}$ & UFV89-334430 & $1,22 \mathrm{ab}$ \\
\hline FT-2003 & $29,64 a$ & BR92-06665 & $43,95 \mathrm{ab}$ & CSP-3 & $1,08 \mathrm{ab}$ \\
\hline CSP-2 & $28,30 \mathrm{ab}$ & CSP-4 & $38,09 \mathrm{ab}$ & BR93-11995 & $1,08 \mathrm{ab}$ \\
\hline BR93-6957 & $26,83 \mathrm{abc}$ & FT-2003 & $32,65 \mathrm{ab}$ & BR92-06665 & $1,05 a b$ \\
\hline UFV89-334430 & $25,65 \mathrm{abcd}$ & UFV89-334430 & $31,06 \mathrm{abc}$ & UFV94- 1896 & $1,00 \mathrm{ab}$ \\
\hline UFV94-1896 & 23,36abcd & FT-2002 & $30,75 a b c$ & FT-20 & $0,92 \mathrm{ab}$ \\
\hline CSP-3 & $23,34 \mathrm{abcd}$ & CSP-2 & $27,88 \mathrm{abcd}$ & CSP-1 & $0,66 \mathrm{ab}$ \\
\hline BR93-11995 & 22,06abcd & CSP-1 & $23,86 \mathrm{abcde}$ & BR93-7411 & $0,47 \mathrm{ab}$ \\
\hline BR93-7411 & 21,44abcde & BR93-11995 & 19,58abcde & OCEPAR-3 & $0,47 \mathrm{ab}$ \\
\hline CSP-4 & 21,09abcde & FT-20 & 19,47abcde & BR93-4854 & $0,25 a b$ \\
\hline UFV94-426803 & 20,91abcde & UFV94-426796 & 16,18 bcde & FT-2002 & $0,25 \mathrm{ab}$ \\
\hline BR92-06665 & 20,79abcde & CSP-3 & 16,01 bcde & CSP-4 & $0,19 \mathrm{ab}$ \\
\hline UFV94-426796 & 18,11abcde & BR93-7411 & 5,59 cde & UFV94-426803 & $0,14 \mathrm{ab}$ \\
\hline CSP-1 & 17,95abcde & BR93-4854 & 4,69 & FT-2003 & $0,06 a b$ \\
\hline OCEPAR-3 & 17,47abcde & CSP-5 & 3,61 & CSP-5 & $0,06 a b$ \\
\hline CSP-5 & 11,20 bcde & BR-16 & 3,12 & BR16 & $0,06 \mathrm{ab}$ \\
\hline BR93-4854 & 10,19 cde & FT-2001 & 2,46 & BR93-6957 & $0,06 a b$ \\
\hline BR-16 & 9,20 & UFV94-426796 & 2,24 & UFV94-426796 & $0,00 \mathrm{~b}$ \\
\hline FT-2001 & 6,55 & UFV94-426803 & 2,06 & FT-2001 & $0,00 \mathrm{~b}$ \\
\hline
\end{tabular}

*Médias seguidas pela mesma letra não diferem entre si, ao nível de 5\% de probabilidade, pelo teste de Tukey. Os dados de incidência foram transformados para arco seno da raiz de x/100 para efeito de análise estatística. 
cou uma menor incidência de $C$. dematium em cultivares de soja provenientes de quatro municípios do Triângulo Mineiro. Em Santa Juliana, as cultivares Savana, UFV-10, IAC-8 e CAC-1, apresentaram, $r$ espectivamente, 0,$3 ; 0,2 ; 0$ e $0 \%$ de incidência de $C$. dematium; em Nova Ponte, para as cultivares Savana, Cristalina e CAC-1, a incidência desse fungo foi de 0,03; 0 e 0\%, respectivamente; em Uberaba, 'Savana', 'UFV10 ' e 'CAC-1' apresentaram uma incidência de 1,7; 0 e $0 \%$, respectivamente; e, em Uberlândia, 'Doko-RC' e 'Cristalina' não foram infetadas pelo patógeno. Este patógeno alcançou grande importância no cerrado brasileiro devido a disseminação pelas sementes. O tratamento químico das sementes, mesmo quando os testes de sanidade acusam baixo índice de incidência do patógeno, poderia ter impedido ou até retardado a sua disseminação entre as áreas produtoras de soja no país (Embrapa, 1996/97).

A germinação correlacionou-se positivamente com vigor das sementes e com a produtividade de grãos $(\mathrm{r}=$ $+0,79$ e $+0,50$, respectivamente), evidenciando que uma melhor população de plantas é a garantia para se obter uma maior produtividade. Observou-se correlação negativa entre as variáveis porcentagem de germinação e a incidência de $F$. semitectum, $P$. sojae e $C$. dematium $(\mathrm{r}=-0,33,-0,39$ e $-0,26$, respectivamente), indicando o efeito desfavorável desses patógenos na germinação das sementes. A variável vigor das sementes correlacionou-se positivamente com a produtividade $(\mathrm{r}=+0,59)$, mas negativamente com a incidência de $F$. semitectum e $P$. sojae $(\mathrm{r}=-0,36$ e $-0,60$, respectivamente), indicando que em programas de melhoramento genético, uma maior atenção deve estar voltada para a realização de testes de sanidade das sementes das linhagens avançadas (sementes genéticas), já que uma alta incidência desses fungos poderá comprometer seriamente o sucesso da futura cultivar, após o seu lançamento a nível nacional.

\section{REFERÊNCIAS BIBLIOGRÁFICAS}

ARANTES, H.A.G., ROCHA, V.S., SILVA, E.A.M. \& SEDIYAMA, T. Espessura do tegumento, embebição em água e qualidade fisiológica da semente de soja. Revista Ceres 41:126-132. 1994.

BANZATTO, D.A \& KRONKA, S.N. Experimentação agrícola. Jaboticabal: FUNEP, 1989.

BRASIL. Ministério da Agricultura. Regras para análise de sementes. Brasília:SNAD/CLAV, 1992.

EMPRESA BRASILEIRA DE PESQUISA AGROPECUÁRIAEMBRAPA. Recomendações técnicas para a cultura da soja na região central do Brasil, 1996/97. Londrina, 1996,

HENNING, A.A. Testes de sanidade de sementes de soja. In: Soave, J. \& Wetzel, M.M.V.S. (Eds.).Patologia de sementes. Campinas, Fundação Cargill. 1987. pp.441-54.

MINAS GERAIS. Secretária de Agricultura e Comércio de estacas, sementes e mudas. Normas, padrões e procedimentos para produção de sementes básicas, certificadas e fiscalizadas. Belo Horizonte, 1996/97.

RESENDE, J.C.F. \& SEDIYAMA,T. Influência do período de armazenamento na germinação e sanidade de sementes de soja. Revista Ceres. 42:575-583, 1995.

SANTOS, V.L.M. Avaliação da germinação e do vigor de sementes de genótipos de soja [Glycine max (L.) Merril], submetidas a stress salino e osmótico. (Tese de Doutorado) Viçosa: Universidade Federal de Viçosa. 1994.

SOUZA, E.R. Avaliação da sanidade de cultivares de soja resistentes ao cancro da haste (Diaphorthe phaseolorum f.sp. meridionalis) com diferentes índices de germinação. (Monografia de conclusão do Curso de Agronomia). Uberlândia, MG: Universidade Federal de Viçosa. 1995.

YORINORI, J.T. Doenças de soja no Brasil. In: A soja no Brasil Central. 3a ed., Campinas: Fundação Cargill, 1986.

YORINORI, J.T., CHARCHAR, M.J.D., NASSER, L.C.B \& HENNING, L.C.B. Doenças de soja e seu controle. In: Arantes, E.A. \& Souza, P.I.M. (Eds.) Cultura da soja nos cerrados. Piracicaba: POTAFOS, 1993. pp.333-397. 